International Journal of Embedded Systems and Applications (IJESA) Vol.2, No.4, December 2012

\title{
A Wireless SENSOR NETWORK BOARD For ENVIRONMENTAL MONITORING USING GNSS AND ANALOG TRIAXIAL ACCELEROMETER
}

\author{
Oscar Rorato ${ }^{1}$, Claudio Lucianaz ${ }^{2}$, Eliana Vittaz ${ }^{3}$, Silvano Bertoldo ${ }^{4}$ \\ Marco Allegretti ${ }^{5}$ and Riccardo Notarpietro ${ }^{6}$
}

${ }^{1}$ DET (Dipartimento di Elettronica e Telecomunicazioni), Politecnico di Torino, Italy oscar.rorato@polito.it

${ }^{2}$ DET (Dipartimento di Elettronica e Telecomunicazioni), Politecnico di Torino, Italy claudio.lucianazepolito.it

${ }^{3}$ Envisens Technologies s.r.l., Quart (AO), Italy

eliana.vittaz@gmail.com

${ }^{4}$ DET (Dipartimento di Elettronica e Telecomunicazioni), Politecnico di Torino, Italy silvano.bertoldo@polito.it

${ }^{5}$ CINFAI (Consorzio Interuniversitario per la Fisica delle Atmosfere e delle Idrosfere), local unit at Politecnico di Torino, Italy marco.allegretti@polito.it

${ }^{6}$ DET (Dipartimento di Elettronica e Telecomunicazioni), Politecnico di Torino, Italy riccardo.notarpietrodpolito.it

\begin{abstract}
Wireless Sensor Networks (WSNs) have attracted an increasing attention in recent years because of the large number of potential applications. They are used for collecting, storing and sharing data, for monitoring applications, surveillance purposes and much more.

On the other hand GNSSs are used in various systems devoted to monitor different atmospheric parameters and to trace displacements of landslides and glaciers in severe environmental conditions and in all weather situations. A first example of low cost DGPS wireless sensor network was installed in 2009 on a serac located at $4100 \mathrm{~m}$ above a populated area in the Aosta Valley, Italy, and it is still operative.

This work presents an evolution of the WSN node used in that systems with improved functionalities and flexibility. The electronic board developed as a multipurpose board to be used in different WSNs, has been completely redesigned as an open system in order to reduce its sizes and to be configured by only varying the firmware on the microcontroller. It allows different interfaces and is equipped with a recovery system, guaranteed by a watchdog chip which continuously monitor the onboard microcontroller.

The board is equipped with both a GNSS module and an analog triaxial accelerometer in order to merge GNSS raw data and accelerometer data to keep track of both fast events and slow events. A free open source operative system has been ported on the microcontroller in order to perform multiple operations and to manage the communications between the network nodes with improved efficiency. The board firmware can be modified in real time using a custom bootloader to avoid difficult maintenance operations.
\end{abstract}

\section{KEYWORDS}

Wireless Sensor Network, DGPS, Electronic Board, Network Nodes, GNSS, Accelerometer 


\section{INTRODUCTION}

A first monitoring system based on a network of single frequency GNSS (Global Navigation Satellite System) receivers was installed on Grandes Jorasses serac in 2009 to trace its displacements [1]. It was made by 3 sensor boards installed over the glacier to acquire GNSS data and 1 collector node to receive and process data installed in a more accessible place.

Due to the inaccessibility of the site and the severe environmental operative conditions, the developed sensors were able to transmit data through a wireless channel from the serac to the collector node ensuring 24 hours a day and all weather conditions monitoring. High measurements accuracy is gained by exploiting the DGPS (Differential Global Positioning System) technique using local permanent GNSS stations.

The drawback of the installation was that the boards were unable to perform multitasking operations: contemporary acquisition of GNSS data and their transmission to the base node was not possible.

To overcome this limitation the network boards have been redesigned starting from a previous developed prototypal multipurpose board [2] considering a more powerful microcontroller unit, more memory storage capacity and adopting an open-source real time operative system to perform multitasking operations. Furthermore a custom wireless protocol has been implemented in order to achieve a more robust communication, and a triaxial analog accelerometer has been installed on the sensor board in order to track fast displacement. A data fusion operation between data collected by GNSS and accelerometers improves the monitoring capabilities of the entire system.

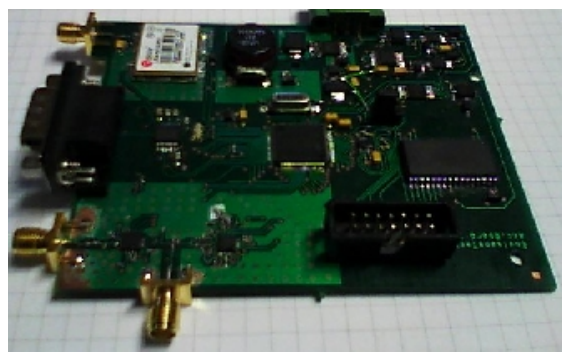

Figure 1. WSN designed board

To make the system more robust, a tool for automatically restart the electronic board in case of "dead lock" of the microcontroller has been included: a watchdog chip which continuously monitors the onboard microcontroller status.

The following sections of the work describe the hardware developed for the new wireless sensor board and its main characteristics, together with some implementation techniques. Two smaller paragraphs are also dedicated to the real time operative system installed on the microcontroller and to the very useful boot loader, realized in order to allow remote firmware update.

\section{BOARD DESCRIPTION AND IMPLEMENTATION TECHNIQUES}

The system consists of four separate sections [3]:

- Analog/digital sensors; 
- $\quad$ Radiofrequency section;

- Microcontroller Unit and memory;

- Power supply stages.

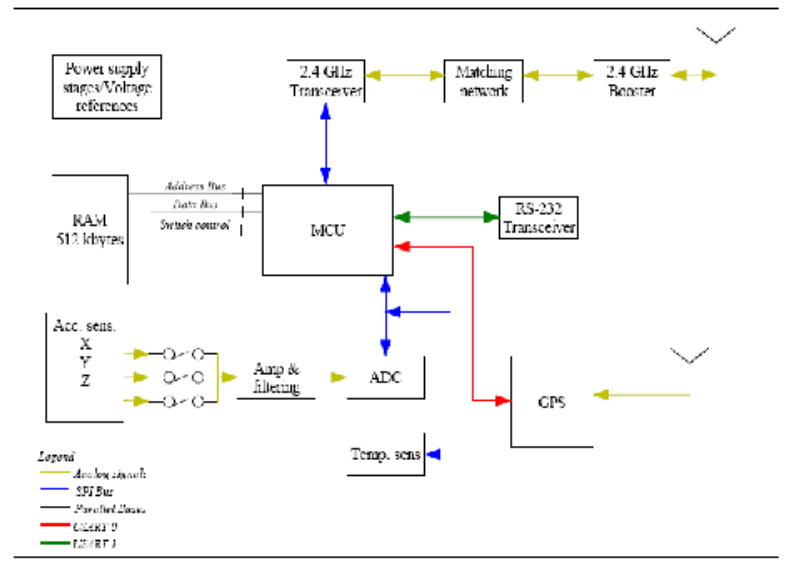

Figure 2. Block diagram of the WSN board

Figure 2 shows the complete block diagram of the electronic board. Its stack-up is a four layers in FR4 substrate. During the layout design phase, particular attention was dedicated to the design of proper RF section (controlled impedance routes, via holes modeling) and to the routing of analog/digital mixed signal section (proper analog/digital grounding techniques, power supply plane).

\subsection{Analog/digital sensors section}

Since its prototypal multipurpose version, the WSN board has been designed to be provided with different types of sensors (e.g. accelerometers, temperature sensors, GNSS, photodiodes). The configuration for the presented WSN board is composed by a GNSS module, a triaxial analog accelerometer and a temperature sensor. Sensors communications are performed via USART or SPI bus.

The used accelerometer is a 16 pin LFCSP package, an analog sensor built with MEMS technology; it has three voltage outputs, one for each coordinate axis and each output has been connected to a conditioning circuit in order to match the input voltage range of the ADC converter. Conditioned outputs are then sent to a 3-ways multiplexer which allow proper selection of each channels during sensor readout.

The GNSS module is a U-Blox LEA-6T. It is configured directly by the WSN board at the start up, acquires satellite data and sends raw data every time a query is performed by the embedded control unit. Data are sampled every 15 seconds ( $\sim 5 \mathrm{MB} /$ day $)$ but the sampling period can be changed in real time remotely sending the appropriate command to the board.

The temperature sensor is fully digital and is used to monitor board's temperature to prevent dangerous overheating.

\subsubsection{Interface design and chip choice}

In order to design the electronic circuits, their interfaces and connections, and to chose the best chip, some simulations have been performed using the software P-Spice ${ }^{\circledR}$. 
The accelerometer has been modeled according to the factory specifications reported on the datasheet of the component (Figure 3) because the model is unavailable on Analog Devices web site[4].

\begin{tabular}{|c|c|c|c|c|c|}
\hline Parameter & Conditions & Min & Typ & Max & Unit \\
\hline $\begin{array}{l}\text { SENSOR INPUT } \\
\text { Measurement Range } \\
\text { Nonlincarity } \\
\text { Package Alignment Error } \\
\text { Interaxis Alignment Error } \\
\text { Cross Axis Sensitivity' }\end{array}$ & $\begin{array}{l}\text { Each axis } \\
\text { Pcrocnt of full scalc }\end{array}$ & \pm 2 & $\begin{array}{l} \pm 2.5 \\
\pm 0.2 \\
11 \\
\pm 0.1 \\
\pm 1\end{array}$ & & $\begin{array}{l}y \\
\% / 6 \\
\text { Degrees } \\
\text { Degrees } \\
\%\end{array}$ \\
\hline $\begin{array}{l}\text { SENSITIVITY (RATIOMETRIC) }{ }^{2} \\
\text { Scnsitivity at X Xur, Your, } Z_{\text {ก }} \\
\text { Sensitivity Change Due to Temperature }{ }^{3}\end{array}$ & $\begin{array}{l}\text { Each axis } \\
V_{5}=3 \mathrm{~V} \\
V_{5}-3 \mathrm{~V}\end{array}$ & 378 & $\begin{array}{l}420 \\
10.01 \\
\end{array}$ & 462 & $\begin{array}{l}\mathrm{mVig} \\
\mathrm{g} / \gamma^{\circ} \mathrm{C}\end{array}$ \\
\hline 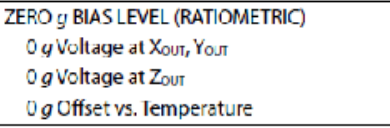 & $\begin{array}{l}V_{5}-3 V \\
V_{5}=3 V\end{array}$ & $\begin{array}{l}1.3 \\
1.2\end{array}$ & $\begin{array}{l}1.5 \\
1.5 \\
11 \\
\end{array}$ & $\begin{array}{l}1.7 \\
1.8\end{array}$ & $\begin{array}{l}\mathrm{v} \\
\mathrm{v} \\
\mathrm{mg} /{ }^{\circ} \mathrm{C}\end{array}$ \\
\hline $\begin{array}{l}\text { NOISE PERFORMANCE } \\
\text { Nolse Density Xout, You, Z Z }\end{array}$ & & & 250 & & $\mu g / \cdot \sqrt{ } \mathrm{Hz} \mathrm{rms}$ \\
\hline $\begin{array}{l}\text { FREQUENCY RESPONSE }{ }^{4} \\
\text { Bandwidth } \mathrm{X}_{\mathrm{OUT}}, \mathrm{Y}_{\mathrm{OUT}^{5}}{ }^{5} \\
\text { Bandwidth } \mathrm{Z}_{\text {OUT }}{ }^{5} \\
\text { RFII Tolerance } \\
\text { Sensor Resunant Frequency }\end{array}$ & $\begin{array}{l}\text { No external filter } \\
\text { No external filter }\end{array}$ & & $\begin{array}{l}1600 \\
550 \\
32 \pm 15 \% \\
5.5 \\
\end{array}$ & & $\begin{array}{l}\mathrm{Hz} \\
\mathrm{Hz} \\
\mathrm{k} \Omega \\
\mathrm{kHz}\end{array}$ \\
\hline
\end{tabular}

Figure 3. Accelerometer specifications (extract from datasheet)

A set of simulations (DC sweep, AC, noise, Monte Carlo, worst case, advanced P-Spice sensitivity) have been performed to design the conditioning circuit: a non-inverting op-amp based amplifier that ensures very high input impedance that avoid sensor loading. The operational amplifier has been chosen taking into account the noise contributions, and its rail-torail output capability. OPA211 has been chose and to reach its best performance it has been powered with dual supply $(+/-12 \mathrm{~V})$.

Since the accelerometer presents three output analog signals coming from each axis, a switch is needed in order to collect data separately from each output. The choice of the TS3A4751 switch is based on the lowest leakage currents criterion.

The signals acquired by ADC converter presents a very low frequency (some tens of hertz). To chose the best converter the most important parameters are the static specification like INL, DNL and offset error: the ADS7279 has been chosen.

To maximize ADC performance, the R-C filter in front of its analog input has been redesigned. In fact the performances of the ADC relate to its internal capacitor that presents a non-linear behavior related to voltage and sampling frequency: to compensate these unwanted behavior we designed an external RC filter [5].

The ADS7279 needs an external voltage reference: REF5050 has been chosen for its high temperature stability and for its very low noise characteristics.

\subsection{Radiofrequency Section}

Each WSN board is equipped with the CC2500 wireless transceiver. It is a low-cost $2.4 \mathrm{GHz}$ transceiver designed for very low power wireless applications. The main operating parameters and the 64-byte transmit/receive FIFOs of CC2500 are controlled via the SPI interface as already mentioned for the analog and digital sensors. 
International Journal of Embedded Systems and Applications (IJESA) Vol.2, No.4, December 2012

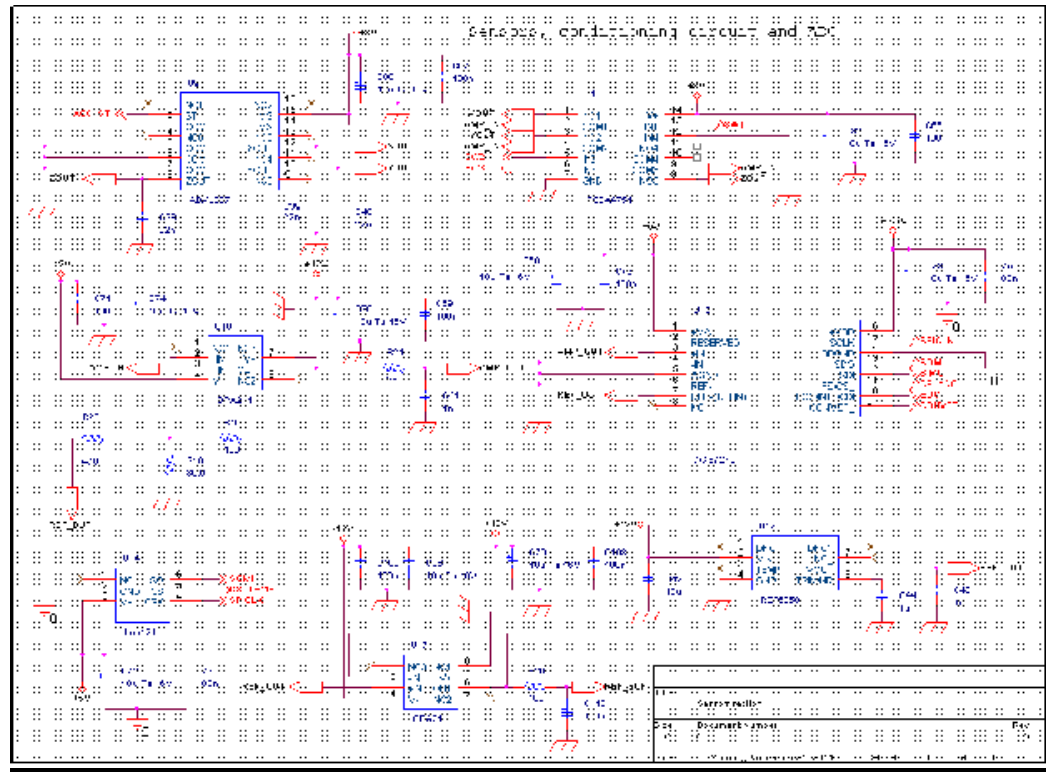

Figure 4. Schematic for sensors interfaces, conditioning circuits and ADC conversion

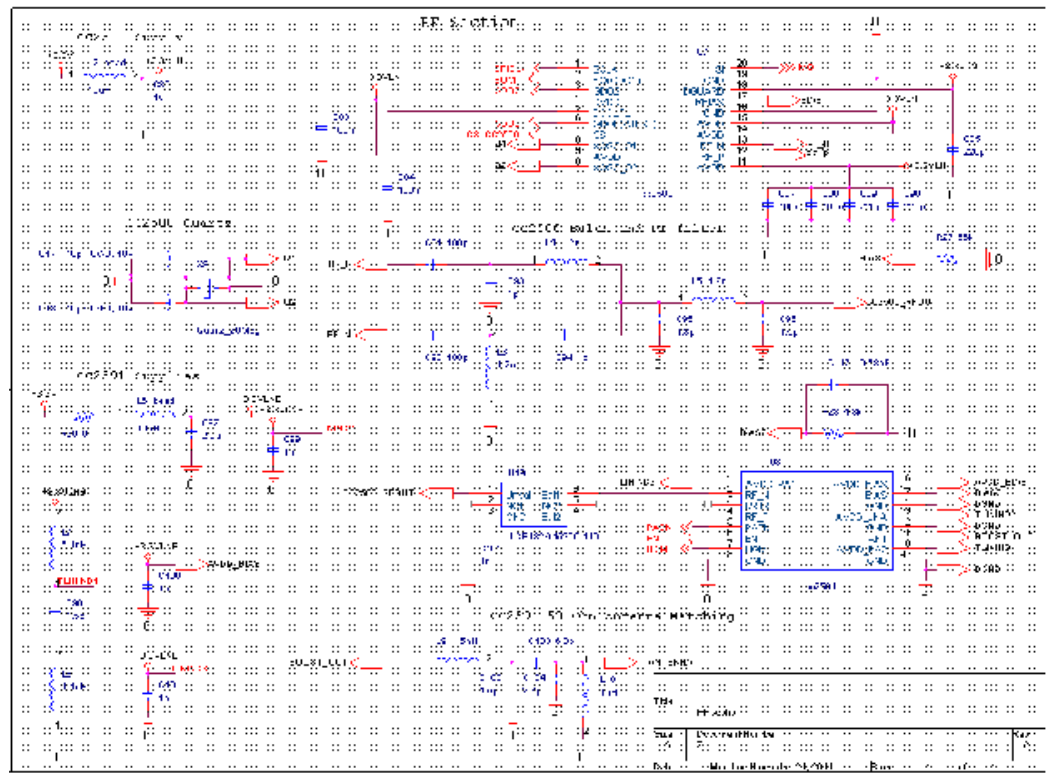

Figure 5. Schematic for the Radiofrequency Section

The other component of the RF section is the CC2591, used both as a low noise amplifier and a power amplifier. The matching between CC2500 and CC2591 has been done according to the following steps:

- using Agilent-ADS ${ }^{\circledR}$ software, the match between CC2500 differential output and the 50 Ohm line has been established;

- the "balun" used in the Texas Instruments CC2591 reference design guide has been used to match the $50 \mathrm{Ohm}$ line to the CC2591 input impedance [6]; 
- the same matching design showed in the Texas Instruments CC2591 reference design guide has been used to match the CC2591 output impedance to the 50 Ohm Antenna.

\subsection{Microcontroller Unit - Memory}

This section of the board presents the core of the system: the microcontroller MSP430F2619. It manages the following components: analog to digital converter, switches, temperature sensor, RF transceivers, memory, GNSS and accelerometer. The MCU also allow the wireless network protocol to run properly.

This type of microcontroller has been chosen due to its low power consumption: when it is used in active mode its current consumption is equal to approximately $8 \mathrm{~mA}$.

The board is equipped with a $512 \mathrm{kB}$ SRAM for data storage and buffering. It is managed as a circular buffer to support multitasking operations. In fact, especially when a radio communication is performed, it is important to continue sensors acquisition in order to avoid data losses.

\subsection{Power supply stages}

The board is powered by a buck switching power supply (TPS54357), which is able to convert the $12 \mathrm{~V}$ positive supply in an output voltage of $5 \mathrm{~V}$. This component has been chosen because it does not require additional external switches and guarantees very low ripple noise, if properly designed. The software SwitcherPRO ${ }^{\circledR}$ is used as an aid to dimension the few external components needed.

The 5 Volt supply is used to feed two LDO (Low Drop Out) regulators, providing 3V (TPS73730) and 3.3V supplies (TPS73733), needed by different components placed on the board. This components have been chosen for their very low quiescent current and very low noise performance.

\section{REAL TIME OPERATIVE SYSTEM}

The first version of the GNSS network was not able to collect GNSS data and transmit them from the sensor to the collector node at the same time: the board did not perform multitasking operations.

In order to make the sensor nodes able to perform various operations at the same time without losing GNSS and accelerometer data, the boards needed to be equipped with a software or a firmware able to operate in a multitasking context.

In particular, the sensor board must perform the following operations:

- Acquire GNSS data

- Acquire accelerometer data

- Store GNSS data in RAM

- Store accelerometer data in RAM

- Acquire data from temperature sensor for board status monitoring

- Radio data transmission

- Wireless network management

As the MCU has a single core architecture it is not possible to perform a hardware multitasking. 
International Journal of Embedded Systems and Applications (IJESA) Vol.2, No.4, December 2012

A Real Time Operative System is one of the best way to perform all the tasks in quasi-real time. The open source FreeRTOS was chosen due to its good performance and due to its open source philosophy and it has been appropriately modified to operate on the board with a proper "porting" operations.

The firmware has been properly realized according to the specifications of the real time operative system, but its description is not the purpose of this work.

\section{A Custom Boot LoAder Realization}

The bootloader is a program on the flash memory of the MCU. It has been designed to communicate through the WSN communication protocol in order to update the firmware on the sensor node.

In fact, when the boards are in the field, severe environmental conditions and their position difficult to reach do not allow to perform ordinary maintenance operations. For instance a firmware update usually performed with the RS232 interface (the common bootloader installed on the MSP430F2619 microcontroller) cannot be done if the boards are installed at more than $4000 \mathrm{~m}$ on a serac to monitor its displacement.

Therefore a new type of wireless bootloader has been realized and has been installed on each sensor board.

In order to update the firmware on the WSN boards the following steps are necessary:

1. Compiling the new firmware in order to obtain an image file in the Texas Instrument proprietary format called "ti-txt";

2. Connect a pc to the access point (which is installed in an accessible place) and send its a command to enable the reception of the new firmware from the pc;

3. The access point sends a request to each sensor nodes in order to put them in "bootloader" mode waiting for its acknowledgement response;

4. The access point sends the "ti-txt" file to the sensor nodes;

5. After the complete reception of the new firmware version the sensor nodes reboot in order to run the new firmware.

\section{CONClusions}

A new wireless sensor network board for environmental monitoring using both GNSS receivers and analog triaxial accelerometer sensors has been described together with its main implementation choices and techniques.

Even if the board is under continuous study and development, the present release is currently installed in a test field, after the good results achieved by the test performed in laboratory.

After the test period this new WSN board will be ready to substitute the ones already installed on Grandes Jorasses serac improving monitoring efficiency.

Other board could also be installed in other different places for environmental operations such as glacier and landslide monitoring. 
International Journal of Embedded Systems and Applications (IJESA) Vol.2, No.4, December 2012

\section{REFERENCES}

[1] Lucianaz C., Rorato O., Allegretti M., Mamino M., Roggero M., Diotri F., (2011) "Low cost DGPS wireless network", IEEE-APS Topical Conference on Antennas and Propagation in Wireless Communications (APWC), 12-16 Sept. 2011, DOI:10.1109/APWC.2011.6046798, pp. 792-795.

[2] Rorato O., Lucianaz C., Bertoldo S., Allegretti M., Perona G., (2012) "A multipurpose node for low cost wireless sensor network, IEEE APWC 2012, Cape Town, South Africa, 2-7 September 2012, pp. 247-250

[3] O. Rorato (2012), Studio e Progettazione di Sistemi Elettronici per Monitoraggio Ambientale Politecnico di Torino, Torino, 2012.

[4] Datasheet (2009), Small, Low Power, 3 Axis \pm 2 g Accelerometer ADXL- 327, www.analog.com Analog Devices

[5] Input Drive Circuitry for SAR ADCs, www.ti.com, Texas Instruments.

[6] Datasheet (2008), CC2691, 2.4-GHz RF Front End, www.ti.com, Texas Instrument

\section{AUTHORS}

Oscar Rorato : He received his degree in Electronic Engineering from Politecnico di Torino, Italy, in 2005. He worked as researcher from 2006 to 2009 in the nanotechnologies field. In 2012 he received the $\mathrm{PhD}$ in Electronic and Communication Engineering at Politecnico di Torino mainly working on FMCW radar design and Wireless Sensor Network design. He is now working on low power electronics and Wireless Sensor Network development.

Claudio Lucianaz : He received his degree in Information Technology Engineering from Politecnico di Torino, Italy, in 2006. In 2010 he receved the $\mathrm{PhD}$ in Electronic and Communication Engineering at Politecnico di Torino mainly working on electromagnetic wave propagation and synthetic aperture radars. He is now working on Wirelesse Sensor Network and GPS monitorin. Moreover, he's working for a foundation dealing with applied research in high mountain where he is responsible of the technical installations and data transmission.

Eliana Vittaz : She received her degree in Computer Science Engineering from Politecnico di Torino, Italy, in 2011. She is now working on firmware development for Wireless Sensor Network for environmental monitoring deployed in alpine environment. She also develops custom applications for smartphone based on spatial localization techniques and GNSS.

Silvano Bertold : He received his degree in Telecommunication engineering from Politecnico di Torino, Italy, in 2008. After some research work concerning forest fire detection, prevention and monitoring started his $\mathrm{PhD}$ program in Electronic and Communication Engineering mainly working on radar systems for meteorology. His research interests also involve Wireless Sensor Network. Keen of sport is member of ISEA (International Sport Engineering Association) since 2010 after an Improvement Course in Sport Engineering attended at Politecnico di Torino.
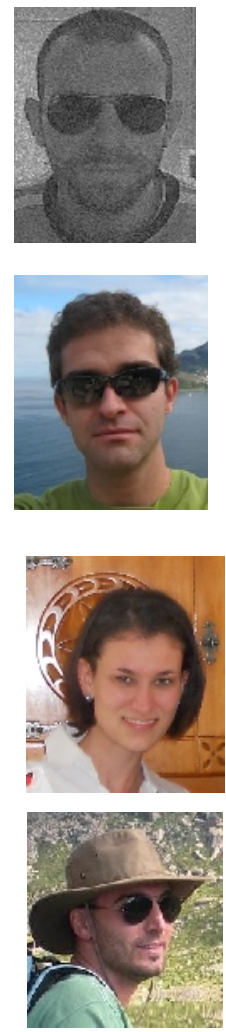
International Journal of Embedded Systems and Applications (IJESA) Vol.2, No.4, December 2012

Marco Allegretti : He received his degree in Electronic Engineering from Politecnico di Torino, Italy, in 2003. In 2005 he attended a Master in Safety Engineering and Risk Analysis and $\mathrm{n} 2007$ he got his $\mathrm{PhD}$ in Electronic and Communication Engineering at Politecnico di Torino. He deals with his various research interests spacing from electromagnetic wave propagation to sensor electronic boards. He is President of "Ordine degli Ingegneri della Provincia di Asti" in charge for the four years 20092013 and he's member of various engineering Italian committees.

Riccardo Notarpietro : Since 2006 Riccardo Notarpietro is assistant professor (in the research area of Electromagnetic Fields) at the Politecnico di Torino. He received his degree in Telecommunications Engineering in 1998 and his PhD in 2001 (Politecnico di Torino) with a thesis on the subject of the atmosphere remote sensing exploiting GPS measurements from space and from ground. His fields of activities included also electromagnetic wave propagation and radar meteorology an. He was involved in several national and international research projects (VOLTAIRE, Software ROSA for

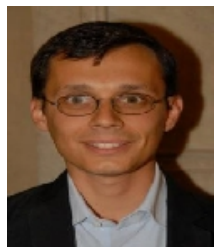
Oceansat-2, METAWAVE. 